1 Identification of a Minimal Peptide Tag for in Vivo and in Vitro Loading

\title{
2 of Encapsulin
}

3 Caleb Cassidy-Amstutz, Luke Oltrogge, Catherine C. Going, Antony Lee, Poh Teng,

4 David Quintanilla, Alexandra East-Seletsky, Evan R. Williams, David F. Savage 


\section{Supplementary Figures}

7 Figure $\mathrm{S} 1$

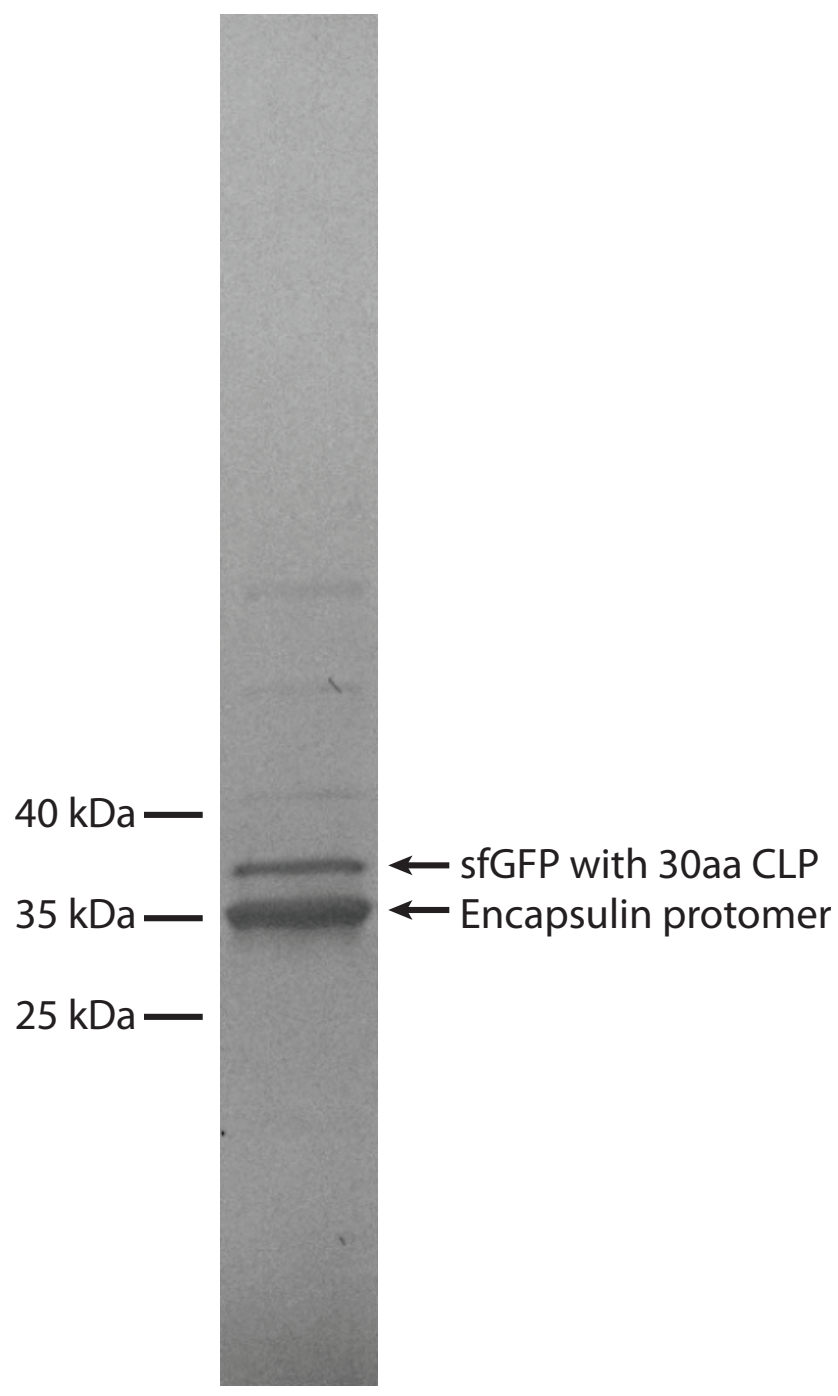

8

9 Figure S1: SDS-PAGE of in vivo purified encapsulin co-expressed with a 30 amino

10 acid CLP tagged sfGFP

11

12

13 
14 Figure S2

A

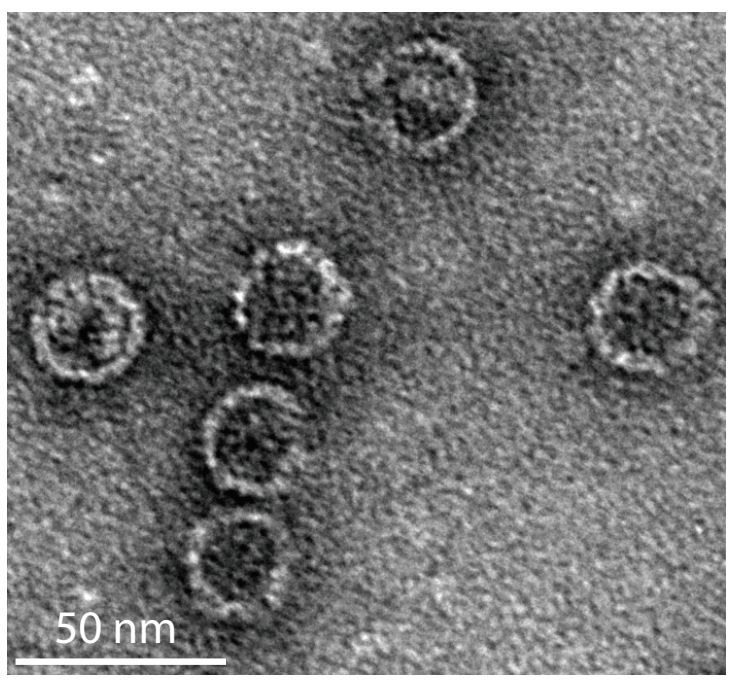

B

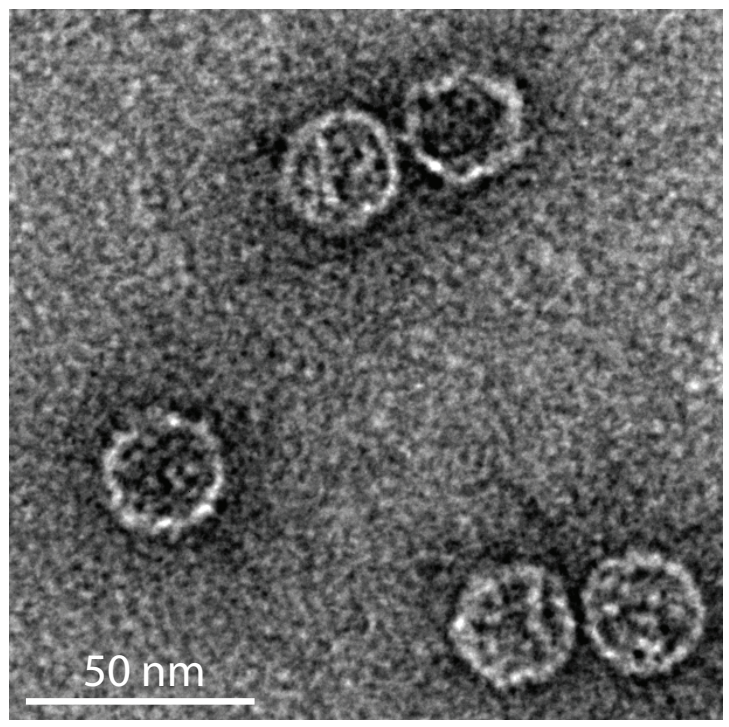

15

16

17 Figure S2: Purified recombinant encapsulins form a nanocompartment. (A)

18 Encapsulin purified from E. coli imaged by TEM. (B) Encapsulin co-expressed with

19 CLP tagged sfGFP purified from $E$. coli imaged by TEM

20 
21 Figure S3

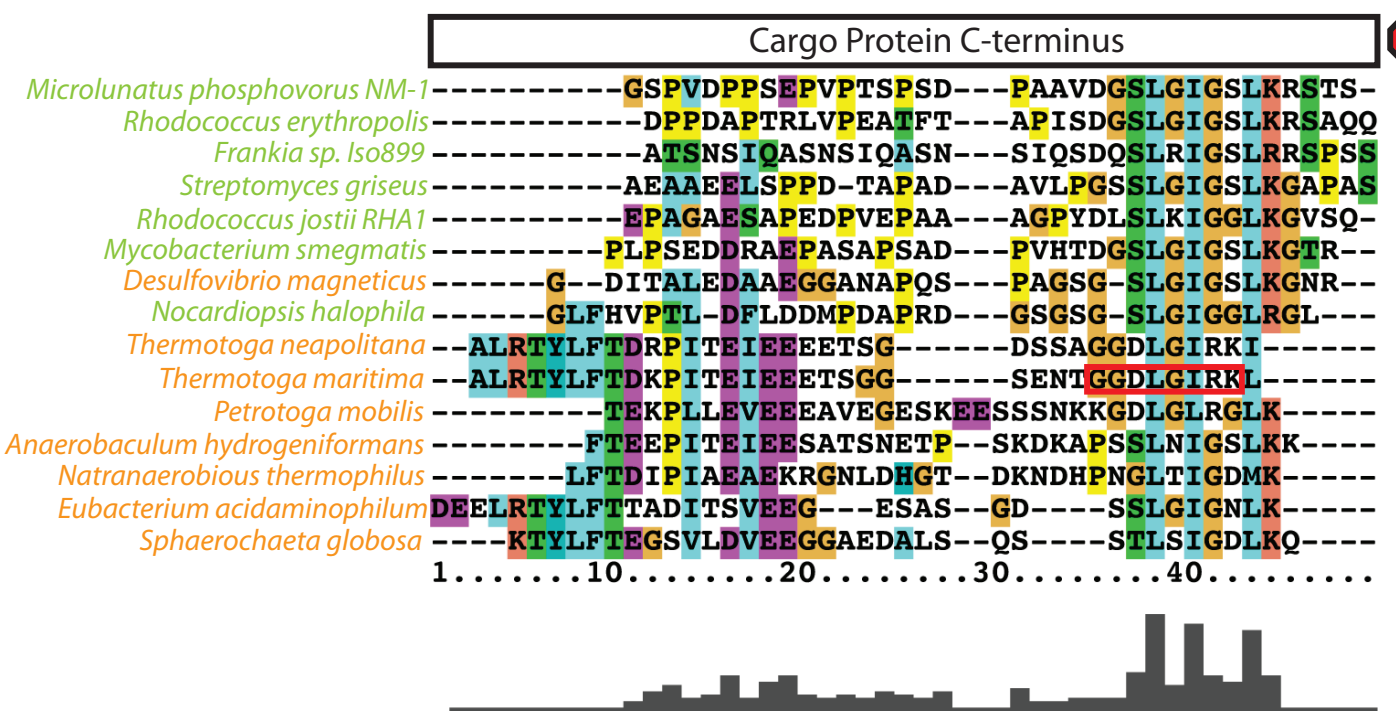

22

23

24 Figure S3: Multiple sequence alignment of CLP sequences from encapsulated DypB

25 and FLP proteins. The peptide found bound to the lumen of T. maritima encapsulin

26 is shown by a red rectangle. Species with an encapsulated DypB are colored in green

27 and species with an encapsulated FLP are colored in orange. 
29 Figure $\mathrm{S} 4$

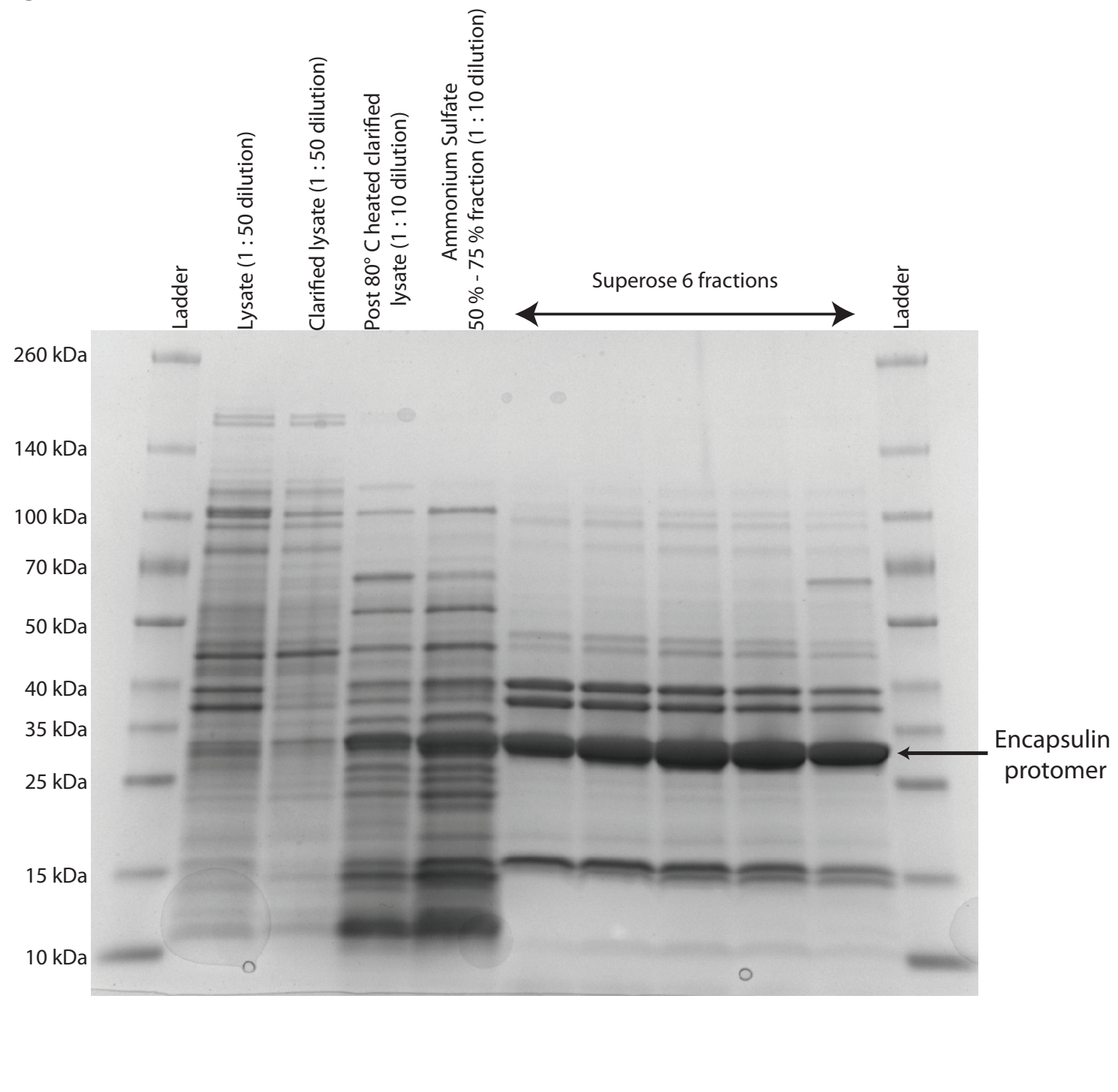

33 Figure S4: SDS-PAGE of the void peak and encapsuiln peak of purified empty

34 encapsulins.

35 
36 Figure 55

A

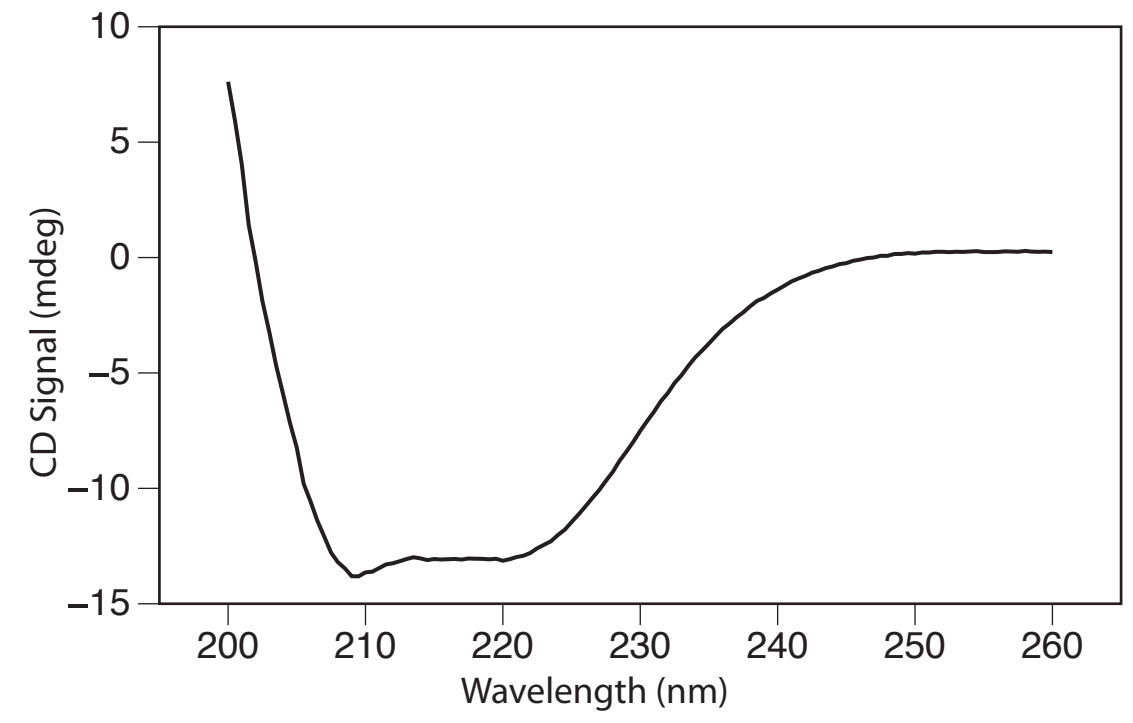

B

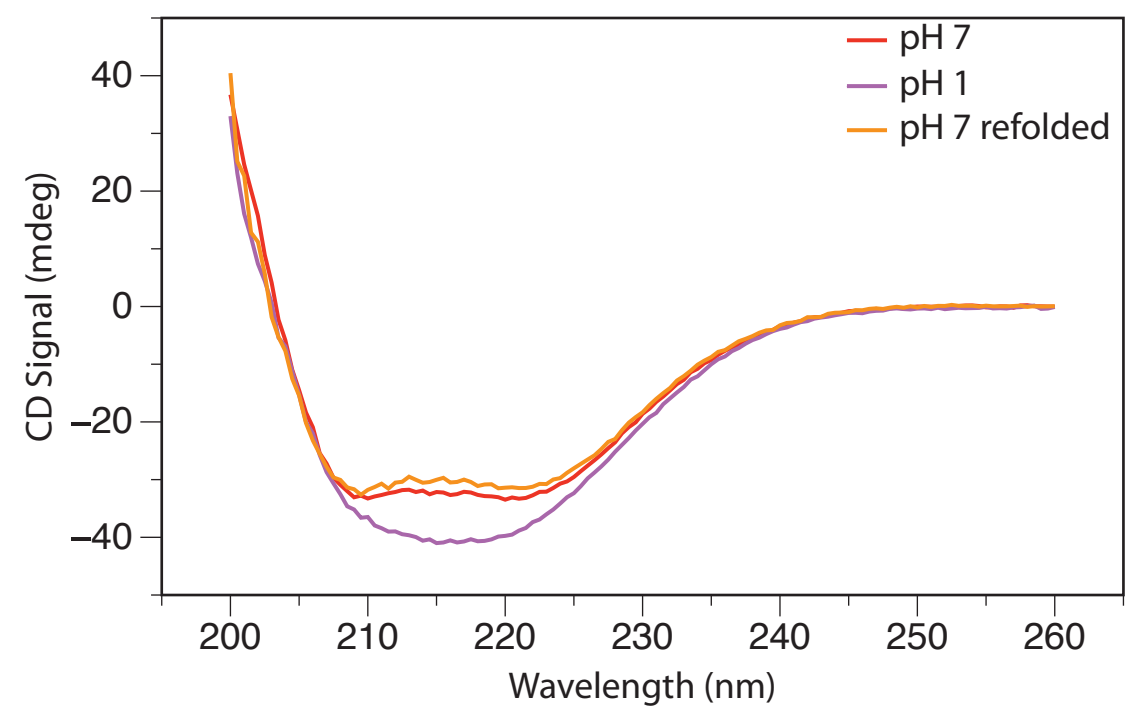

39 Figure S5: CD spectra of encapsulin (A) Spectra of purified empty encapsulin at $\mathrm{pH}$

407.4 (B) Overlay of the CD spectrum of empty encapsulins before acid disassembly, at

$41 \mathrm{pH} \mathrm{1,} \mathrm{and} \mathrm{following} \mathrm{reassembly}$ 
Figure S6
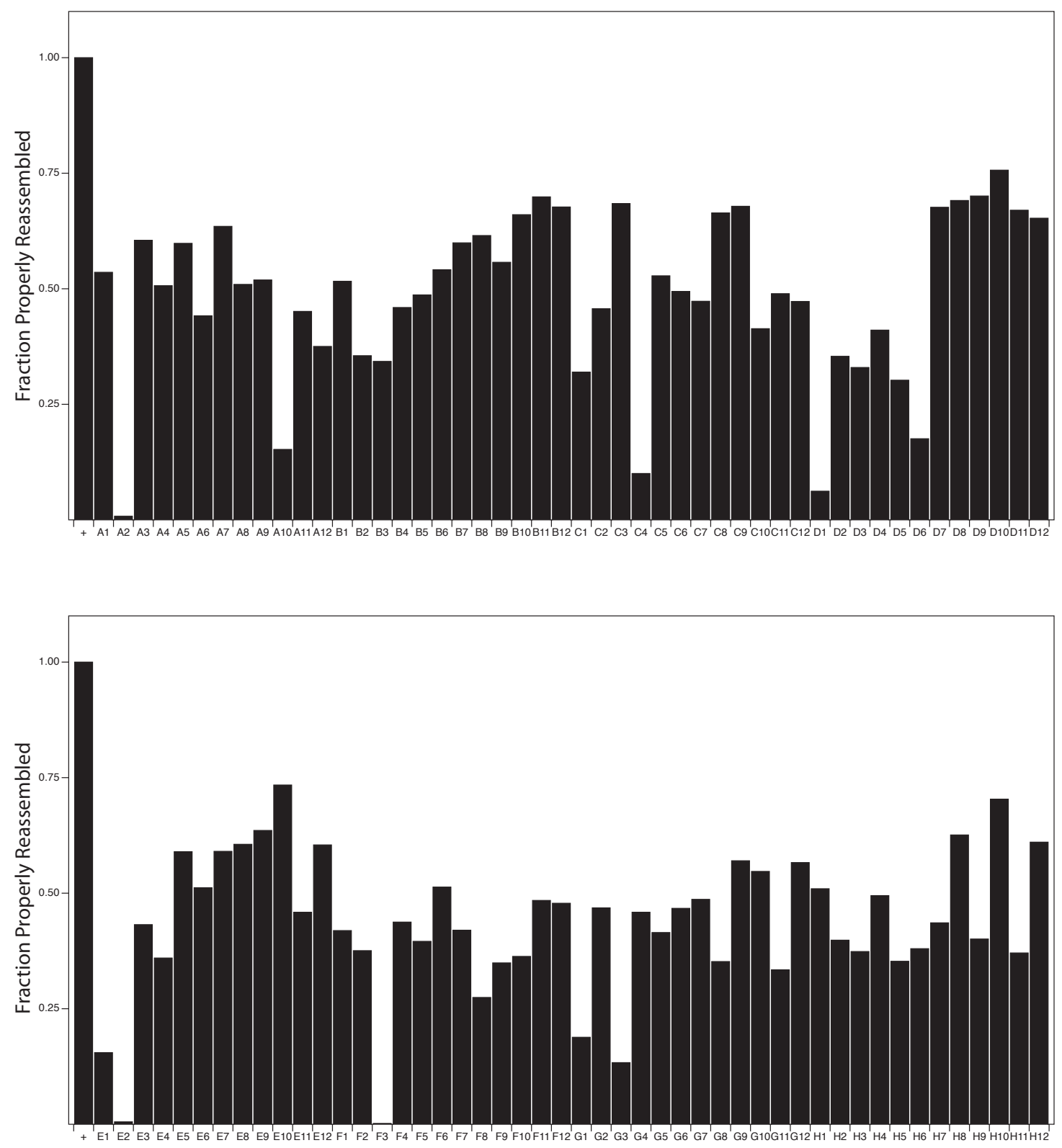

46 Figure S6: Additive Screen to Improve Reassembly Efficiency. Empty encapsulin was

47 disassembled by $\mathrm{GuHCl}$ and reassembled by 10 -fold dilution into buffer containing

48 the additive. The (+) sample was not disassembled and served as the reference

49 point for complete reassembly. The additive screen for each condition is listed in

$50 \quad$ Supplementary Table 3. 
$51 \quad$ Figure S7

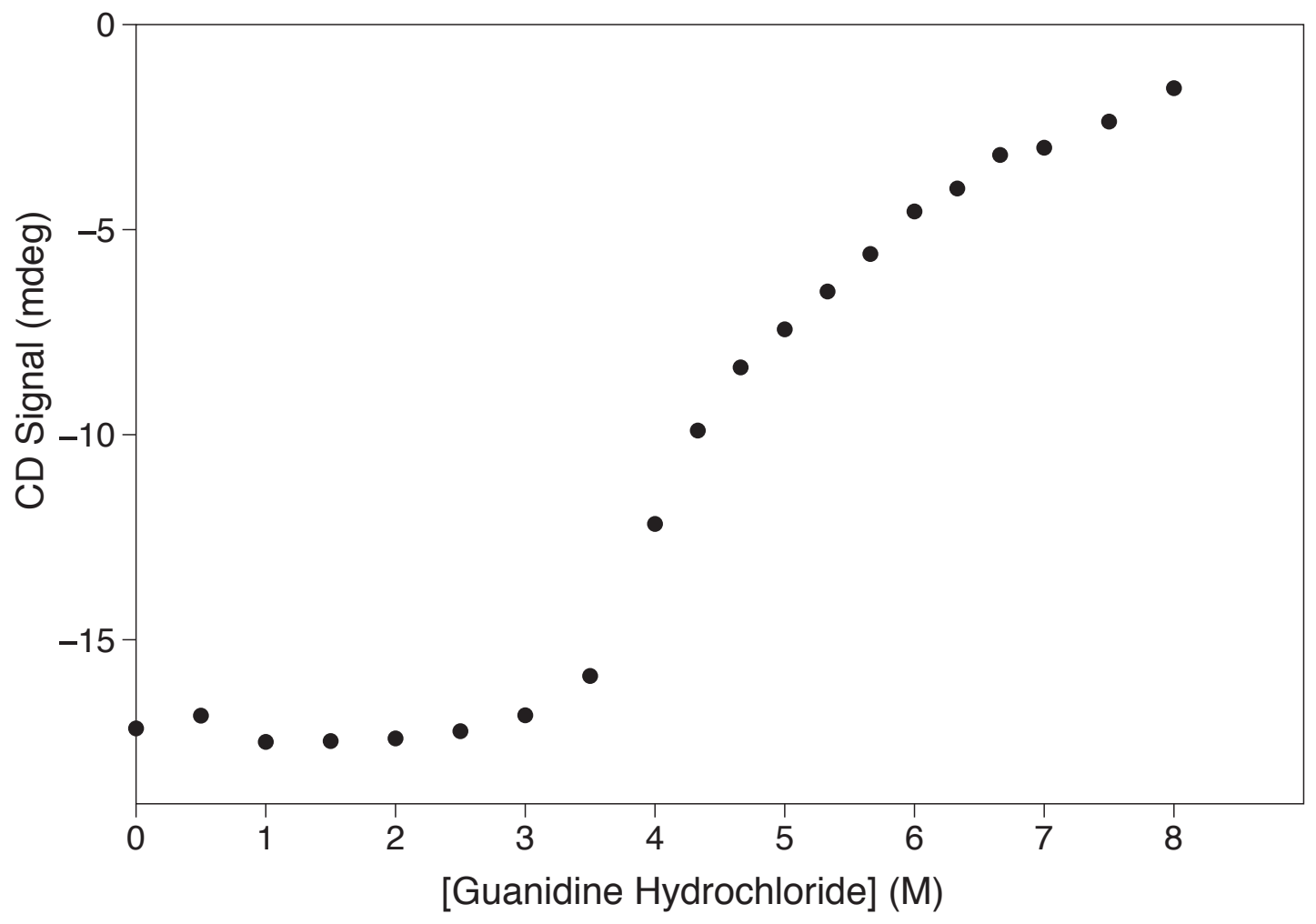

52

53

54 Figure S7: Equilibrium guanidine hydrochloride melt curve of empty encapsulin.

55

56 
$57 \quad$ Figure S8

6X His tag
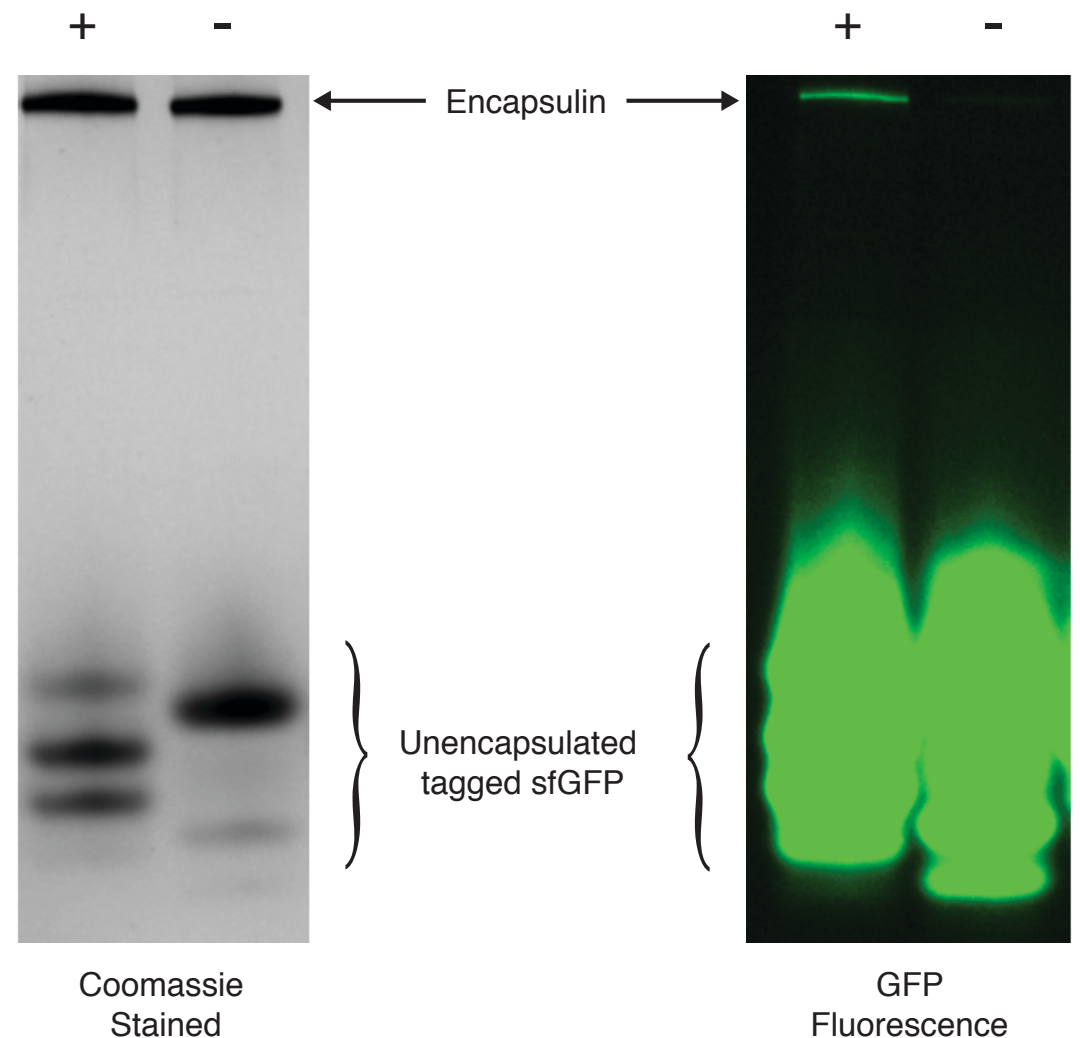

58

59

60 Figure S8: A hexahistidine affinity tag nonspecifically interacts with encapsulin.

61 Coomassie stained (left) and GFP fluorescence (right) from native PAGE of

62 encapsulin incubated with CLP tagged sfGFP that either has or lacks a hexahistidine

63 affinity tag.

64 
65 Figure $S 9$

A

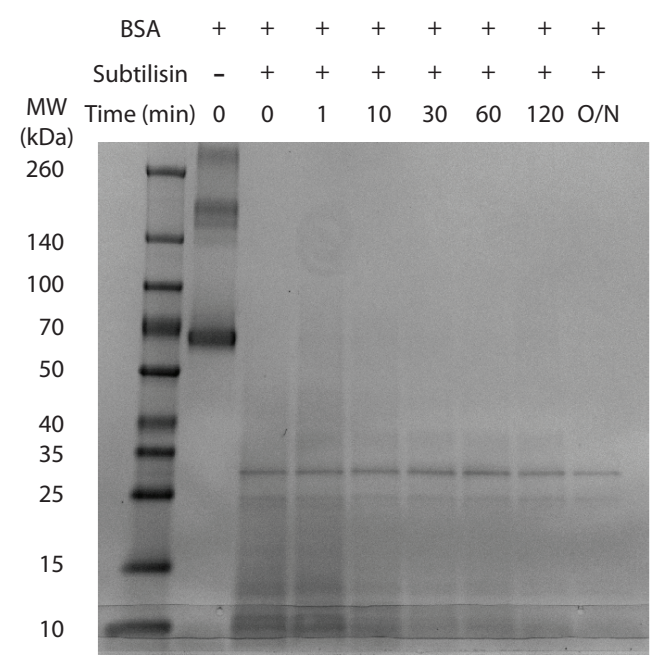

C

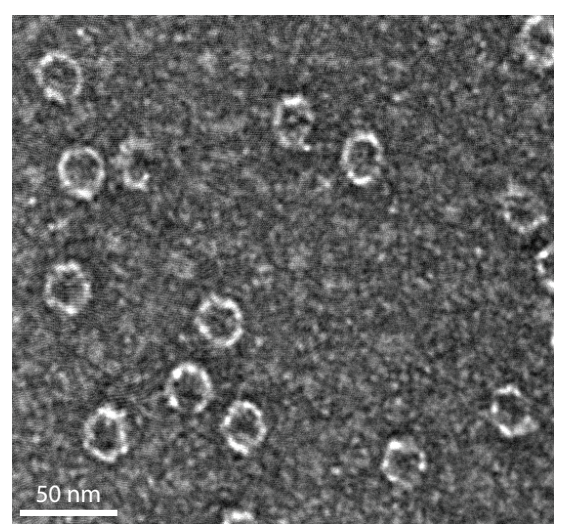

B

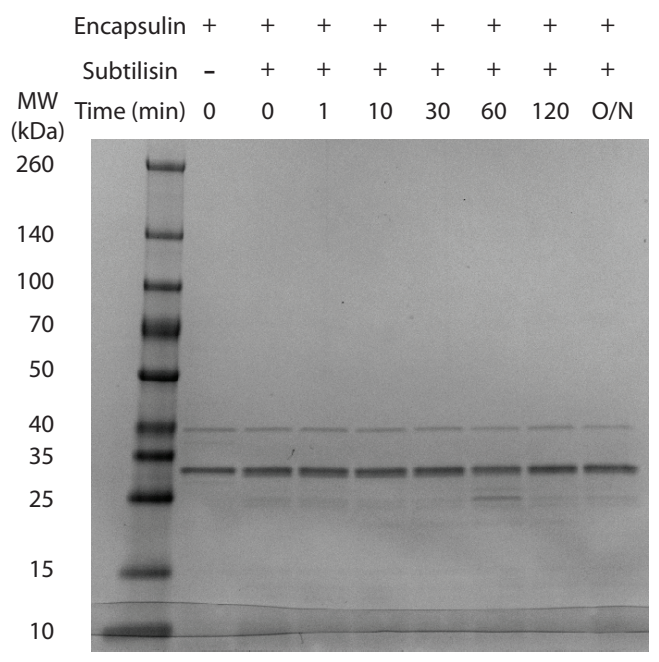

D

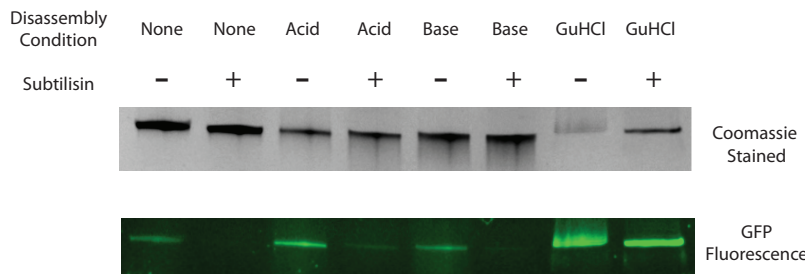

68 Figure S9: Encapsulin is highly resistant to proteases. (A) SDS-PAGE of bovine serum

69 albumin treated with subtilisin. (B) SDS-PAGE of encapsulin treated with subtilisin.

70 (C) TEM of encapsulin after 48 hours of subtilisin treatment. (D) Coomassie and GFP

71 fluorescence from native PAGE of empty encapsulin in vitro loaded with a 30 amino

72 acid CLP following treatment with subtilisin. 


\section{Supplementary Tables}

75

76 Table 1: Plasmids used in this manuscript

\begin{tabular}{|c|l|}
\hline Plasmid Name & \multicolumn{1}{c|}{ Description } \\
\hline pDEST & pET 14 based Golden Gate destination plasmid \\
pDEST-Nhis & pET 14 based Golden Gate destination plasmid with a N terminal 6X His tag \\
pDEST-Chis & pET 14 based Golden Gate destination plasmid with a C terminal 6X His tag \\
pGFP30_Encap & Operon of sfGFP with a 30 amino acid CLP and Encapsulin in pDEST-Chis \\
pGFP15_Encap & Operon of sfGFP with a 15 amino acid CLP and Encapsulin in pDEST-Chis \\
pGFP5_Encap & Operon of sfGFP with a 5 amino acid CLP and Encapsulin in pDEST-Chis \\
pGFP_Encap & Operon of sfGFP with no CLP and Encapsulin in pDEST-Chis \\
pGFPRand_Encap & Operon of sfGFP with a randomized 30 amino acid CLP and Encapsulin in pDEST-Chis \\
pEmpty_Encap & Encapsulin in pDEST \\
pGFP30 & sfGFP with a 30 amino acid CLP in pDEST-Nhis \\
pGFP15 & sfGFP with a 15 amino acid CLP in pDEST-Nhis \\
pGFP5 & sfGFP with a 5 amino acid CLP in pDEST-Nhis \\
pGFP & sfGFP with no CLP in pDEST-Nhis \\
pGFPRand & sfGFP with a randomized 30 amino acid CLP in pDEST-Nhis
\end{tabular}

Table 2: Primers used in this manuscript

82

83

\begin{tabular}{|c|l|}
\hline Primer Name & \multicolumn{1}{|c|}{ Primer Sequence } \\
\hline Encap_f & catgacGGTCTCTgaggaacaatATGAGCGAGTTTCTGAAACGCA \\
Encap_r & catgacGGTCTCTcgctGAACTTCAGCAGGATCAGGG \\
FLP_30aa_tag_f & cacaccGGTCTCCCAAAgaagaagaaacgtccgg \\
FLP_30aa_tag_r & catgacGGTCTCTcctcCAATtcagagcttccttatgccgagg \\
sfGFP_f & catgacGGTCTCTGTCCAAAGGTGAAGAACTGTTCACCGGTGTTG \\
SfGFP_r & catgacGGTCTCTtttgtagagctcatccatgccgt \\
FLP_15aa_tag_f & catgacggtctctcaaaggtggatcagaaaacacgg \\
FLP_5aa_tag_f & catgacggtctctcaaaggcataaggaagctctgaATTG \\
FLP_0aa_ttag_f & catgacggtctctcaaaATTGgaggaacaatATGAGCGAG \\
Empty_Encap_f & catgacGGTCTCTgtccGAGTTTCTGAAACGCAGCTTCGC \\
Encap_removal_r & catgacGGTCTCTcgctCGCTCCTattgttcctcCAATtca \\
\hline
\end{tabular}

84

85 


\section{Table 3: Additives Screened to Improve Reassembly Efficiency}

\begin{tabular}{|c|c|c|c|c|c|c|}
\hline Well & [conc] & units & Additive 1 & [conc] & units & Additive 2 \\
\hline A01 & 10 & $\% \mathrm{v} / \mathrm{v}$ & Water & & & \\
\hline $\mathrm{A} 02$ & 7.5 & $\% w / v$ & Trichloroacetic acid & & & \\
\hline A03 & 25 & $\mathrm{mM}$ & L-Arginine & & & \\
\hline A04 & 25 & $\mathrm{mM}$ & L-Arginine & 25 & $\mathrm{mM}$ & L-Glutamic acid \\
\hline A05 & 50 & $\mathrm{mM}$ & Glycine & & & \\
\hline A06 & 50 & $\mathrm{mM}$ & L-Proline & & & \\
\hline $\mathrm{A} 07$ & 12 & $\mathrm{mM}$ & L-Histidine & & & \\
\hline A08 & 50 & $\mathrm{mM}$ & $\beta$-Alanine & & & \\
\hline A09 & 50 & $\mathrm{mM}$ & L-Serine & & & \\
\hline A10 & 50 & $\mathrm{mM}$ & L-Arginine ethyl ester dihydrochloride & & & \\
\hline A11 & 50 & $\mathrm{mM}$ & L-Argininamide dihydrochloride & & & \\
\hline A12 & 50 & $\mathrm{mM}$ & 6-Aminohexanoic acid & & & \\
\hline B01 & 50 & $\mathrm{mM}$ & Gly-gly & & & \\
\hline B02 & 20 & $\mathrm{mM}$ & Gly-gly-gly & & & \\
\hline B03 & 0.5 & $\% w / v$ & Tryptone & & & \\
\hline B04 & 250 & $\mathrm{mM}$ & Betaine monohydrate & & & \\
\hline B05 & 75 & $\mathrm{mM}$ & D-(+)-Trehalose dihydrate & & & \\
\hline B06 & 200 & $\mathrm{mM}$ & Xylitol & & & \\
\hline B07 & 200 & $\mathrm{mM}$ & D-Sorbitol & & & \\
\hline B08 & 200 & $\mathrm{mM}$ & Sucrose & & & \\
\hline B09 & 50 & $\mathrm{mM}$ & Hydroxyectoine & & & \\
\hline B10 & 250 & $\mathrm{mM}$ & Trimethylamine $\mathrm{N}$-oxide dihydrate & & & \\
\hline B11 & 200 & $\mathrm{mM}$ & Methyl- $\alpha$-D-glucopyranoside & & & \\
\hline B12 & 1 & $\% \mathrm{v} / \mathrm{v}$ & Triethylene glycol & & & \\
\hline $\mathrm{C} 01$ & 50 & $\mathrm{mM}$ & Spermine tetrahydrochloride & & & \\
\hline $\mathrm{CO} 2$ & 50 & $\mathrm{mM}$ & Spermidine & & & \\
\hline $\mathrm{CO3}$ & 50 & $\mathrm{mM}$ & 5-Aminovaleric acid & & & \\
\hline $\mathrm{CO4}$ & 50 & $\mathrm{mM}$ & Glutaric acid & & & \\
\hline $\mathrm{CO5}$ & 8 & $\mathrm{mM}$ & Adipic acid & & & \\
\hline C06 & 50 & $\mathrm{mM}$ & Ethylenediamine dihydrochloride & & & \\
\hline $\mathrm{CO7}$ & 50 & $\mathrm{mM}$ & Guanidine hydrochloride & & & \\
\hline $\mathrm{C08}$ & 50 & $\mathrm{mM}$ & Urea & & & \\
\hline C09 & 50 & $\mathrm{mM}$ & N-Methylurea & & & \\
\hline C10 & 20 & $\mathrm{mM}$ & N-Ethylurea & & & \\
\hline C11 & 3 & $\% w / v$ & N-Methylformamide & & & \\
\hline C12 & 0.3 & $\% w / v$ & Hypotaurine & & & \\
\hline D01 & 15 & $\mathrm{mM}$ & TCEP hydrochloride & & & \\
\hline D02 & 2 & $\mathrm{mM}$ & GSH (L-Glutathione reduced) & 2 & $\mathrm{mM}$ & GSSG (L-Glutathione oxidized) \\
\hline D03 & 0.5 & $\% w / v$ & Benzamidine hydrochloride & & & \\
\hline D04 & 5 & $\mathrm{mM}$ & $\begin{array}{l}\text { Ethylenediaminetetraacetic acid disodium salt } \\
\text { dihydrate }\end{array}$ & & & \\
\hline D05 & 10 & $\mathrm{mM}$ & Magnesium chloride hexahydrate & 10 & $\mathrm{mM}$ & Calcium chloride dihydrate \\
\hline D06 & 10 & $\mathrm{mM}$ & Cadmium chloride hydrate & 10 & $\mathrm{mM}$ & Cobalt(II) chloride hexahydrate \\
\hline D07 & 100 & $\mathrm{mM}$ & Non Detergent Sulfobetaine 195 (NDSB-195) & & & \\
\hline
\end{tabular}




\begin{tabular}{|c|c|c|c|c|c|c|}
\hline D08 & 100 & $\mathrm{mM}$ & Non Detergent Sulfobetaine 201 (NDSB-201) & & & \\
\hline D09 & 100 & $\mathrm{mM}$ & Non Detergent Sulfobetaine 211 (NDSB-211) & & & \\
\hline D10 & 100 & $\mathrm{mM}$ & Non Detergent Sulfobetaine 221 (NDSB-221) & & & \\
\hline D11 & 80 & $\mathrm{mM}$ & Non Detergent Sulfobetaine 256 (NDSB-256) & & & \\
\hline D12 & 50 & $\mathrm{mM}$ & Taurine & & & \\
\hline E01 & 50 & $\mathrm{mM}$ & Acetamide & & & \\
\hline E02 & 50 & $\mathrm{mM}$ & Oxalic acid dihydrate & & & \\
\hline E03 & 50 & $\mathrm{mM}$ & Sodium malonate $\mathrm{pH} 7.0$ & & & \\
\hline E04 & 50 & $\mathrm{mM}$ & Succinic acid pH 7.0 & & & \\
\hline E05 & 0.5 & $\% \mathrm{v} / \mathrm{v}$ & Tacsimate $\mathrm{pH} 7.0$ & & & \\
\hline E06 & 2.5 & $\% \mathrm{w} / \mathrm{v}$ & Tetraethylammonium bromide & & & \\
\hline E07 & 2.5 & $\% \mathrm{w} / \mathrm{v}$ & Cholin acetate & & & \\
\hline E08 & 2.5 & $\% \mathrm{w} / \mathrm{v}$ & 1-Ethyl-3-methylimidazolium acetate & & & \\
\hline E09 & 2.5 & $\% \mathrm{w} / \mathrm{v}$ & 1-Butyl-3-methylimidazolium chloride & & & \\
\hline E10 & 2.5 & $\% \mathrm{w} / \mathrm{v}$ & Ethylammonium nitrate & & & \\
\hline E11 & 50 & $\mathrm{mM}$ & Ammonium sulfate & & & \\
\hline E12 & 50 & $\mathrm{mM}$ & Ammonium chloride & & & \\
\hline F01 & 50 & $\mathrm{mM}$ & Magnesium sulfate hydrate & & & \\
\hline F02 & 50 & $\mathrm{mM}$ & Potassium thiocyanate & & & \\
\hline F03 & 25 & $\mathrm{mM}$ & Gadolinium(III) chloride hexahydrate & & & \\
\hline F04 & 25 & $\mathrm{mM}$ & Cesium chloride & & & \\
\hline F05 & 25 & $\mathrm{mM}$ & 4-Aminobutyric acid (GABA) & & & \\
\hline F06 & 50 & $\mathrm{mM}$ & Lithium nitrate & & & \\
\hline F07 & 50 & $\mathrm{mM}$ & DL-Malic acid pH 7.0 & & & \\
\hline F08 & 50 & $\mathrm{mM}$ & Lithium citrate tribasic tetrahydrate & & & \\
\hline F09 & 25 & $\mathrm{mM}$ & Ammonium acetate & & & \\
\hline F10 & 25 & $\mathrm{mM}$ & Sodium benzenesulfonate & & & \\
\hline F11 & 25 & $\mathrm{mM}$ & Sodium $\mathrm{p}$-toluenesulfonate & & & \\
\hline $\mathrm{F} 12$ & 100 & $\mathrm{mM}$ & Sodium chloride & & & \\
\hline G01 & 140 & $\mathrm{mM}$ & Potassium chloride & & & \\
\hline G02 & 70 & $\mathrm{mM}$ & Sodium phosphate monobasic monohydrate & 130 & $\mathrm{mM}$ & Potassium phosphate dibasic \\
\hline G03 & 100 & $\mathrm{mM}$ & Sodium sulfate decahydrate & & & \\
\hline G04 & 140 & $\mathrm{mM}$ & Lithium chloride & & & \\
\hline G05 & 100 & $\mathrm{mM}$ & Sodium bromide & & & \\
\hline G06 & 4 & $\% v / v$ & Glycerol & 40 & $\mathrm{mM}$ & Lithium chloride \\
\hline G07 & 5 & $\% v / v$ & Glycerol & & & \\
\hline G08 & 1 & $\% \mathrm{v} / \mathrm{v}$ & Ethylene glycol & & & \\
\hline G09 & 1 & $\% \mathrm{v} / \mathrm{v}$ & Polyethylene glycol 200 & & & \\
\hline G10 & 0.5 & $\% v / v$ & Polyethylene glycol monomethyl ether 550 & & & \\
\hline G11 & 0.5 & $\% \mathrm{w} / \mathrm{v}$ & Polyethylene glycol monomethyl ether 750 & & & \\
\hline G12 & 5 & $\% \mathrm{v} / \mathrm{v}$ & Formamide & & & \\
\hline H01 & 5 & $\% \mathrm{v} / \mathrm{v}$ & Polypropylene glycol P 400 & & & \\
\hline H02 & 2.5 & $\% v / v$ & Pentaerythritol ethoxylate $(15 / 4 \mathrm{EO} / \mathrm{OH})$ & & & \\
\hline H03 & 1 & $\% \mathrm{w} / \mathrm{v}$ & 1,2-Propanediol & & & \\
\hline HO4 & 0.3 & $\% \mathrm{w} / \mathrm{v}$ & Polyethylene glycol monomethyl ether 1,900 & & & \\
\hline H05 & 0.3 & $\% \mathrm{w} / \mathrm{v}$ & Polyethylene glycol 3,350 & & & \\
\hline
\end{tabular}




$$
\begin{array}{rrll}
\text { H06 } & 0.3 & \% \text { w/v } & \text { Polyethylene glycol 8,000 } \\
\text { H07 } & 0.2 & \% \text { w/v } & \text { Polyvinylpyrrolidone K15 } \\
\text { H08 } & 10 & \mathrm{mM} & \text { 6-O- } \alpha \text {-D-Maltosyl- } \beta \text {-cyclodextrin } \\
\text { H09 } & 1 & \mathrm{mM} & \text { (2-Hydroxypropyl)- } \beta \text {-cyclodextrin } \\
\mathrm{H} 10 & 8 & \mathrm{mM} & \alpha \text {-Cyclodextrin } \\
\mathrm{H} 11 & 1 & \mathrm{mM} & \beta \text {-Cyclodextrin } \\
\mathrm{H} 12 & 5 & \mathrm{mM} & \text { Methyl- } \beta \text {-cyclodextrin }
\end{array}
$$

\section{Supplementary Material and Methods:}

91 Multiple Sequence Alignment

92 A BLAST search of the non-redundant database against T. maritima FLP

93 sequence revealed other encapsulated FLP proteins. Encapsulated DypB sequences

94 were identified similarly. The multiple sequence alignment was generated and

95 viewed with Clustal $X^{29}$.

97 Reassembly Additive Screen

$9820 \mu \mathrm{M}$ empty encapsulin was disassembled in $7 \mathrm{M} \mathrm{GuHCl}$. Refolding was 99 initiated by diluting 10 -fold into buffer containing the additive. Additives were 100 taken from the Solublity \& Stability Screen (Hampton Research, Aliso Viejo CA).

102 Protease Sensitivity

$1032 \mu \mathrm{M}$ empty encapsulin or $2 \mu \mathrm{M}$ bovine serum albumin (BSA) (Sigma-Aldrich)

104 was incubated with $5 \mu \mathrm{g}$ of subtilisin for the indicated time at RT. The reaction was 105 stopped by addition of 4 X SDS loading dye and heating at $90^{\circ} \mathrm{C}$ for 10 minutes. For 106 TEM, a sample was taken after 48 hours at RT and imaged by negative stain. 
108 Removal of the hexahistidine affinity tag

109 Purified hexahistidine tagged sfGFP was incubated with $100: 1(\mathrm{w} / \mathrm{w})$

110 protease from the tobacco etch virus overnight at $4^{\circ} \mathrm{C}$. Protease and uncleaved

111 sfGFP was removed by running the reaction over Ni-NTA resin equilibrated with

112 buffer A. The flow-through was concentrated using a Vivaspin concentrator.

113

114 References

115 (29) Larkin, M. A., Blackshields, G., Brown, N. P., Chenna, R., McGettigan, P. A.,

116 McWilliam, H., Valentin, F., Wallace, I. M., Wilm, A., Lopez, R., Thompson, J. D., Gibson,

117 T. J., and Higgins, D. G. (2007) Clustal W and Clustal X version 2.0. Bioinformatics 23,

$118 \quad 2947-2948$. 